\title{
Concurrent J-Evolving Refocusing Pulses
}

\author{
Sebastian Ehni ${ }^{1,2,3}$, Martin R. M. Koos ${ }^{1,4,5}$, Tony Reinsperger ${ }^{1,2,3}$, Jens D. Haller ${ }^{1,2}$, David L. Goodwin ${ }^{1,6}$, \\ and Burkhard Luy ${ }^{1,2}$ \\ ${ }^{1}$ Institute for Biological Interfaces 4 - Magnetic Resonance, Karlsruhe Institute of Technology (KIT), Karlsruhe, Germany \\ ${ }^{2}$ Institute of Organic Chemistry, Karlsruhe Institute of Technology (KIT), Karlsruhe, Germany \\ ${ }^{3}$ Bruker Biospin GmbH: Ettlingen 76275, Germany; Fällanden 8117, Switzerland \\ ${ }^{4}$ Pfizer Inc.: Groton, Connecticut 06340, USA \\ ${ }^{5}$ Department of Chemistry, Carnegie Mellon University, Pittsburgh, USA \\ ${ }^{6}$ Chemistry Research Laboratory, University of Oxford, Mansfield Road, Oxford, OX1 3TA, UK \\ Correspondence: David L. Goodwin (david.goodwin@partner.kit.edu) and Burkhard Luy (burkhard.luy@kit.edu)
}

\begin{abstract}
Conventional refocusing pulses are optimised for a single spin without considering any type of coupling. However, despite the fact that most couplings will result in undesired distortions, refocusing in delay-pulse-delay-type sequences with desired heteronuclear coherence transfer might be enhanced considerably by including coupling evolution into pulse design. We provide a proof of principle study for a Hydrogen-Carbon refocusing pulse sandwich with inherent J-evolution following the previously reported ICEBERG-principle with improved performance in terms of refocusing performance and/or overall effective coherence transfer time. Pulses are optimised using optimal control theory with a newly derived quality factor and zcontrols as an efficient tool to speed up calculations. Pulses are characterised in detail and compared to conventional concurrent refocusing pulses, clearly showing an improvement for the J-evolving pulse sandwich. As a side-product, also efficient Jcompensated refocusing pulse sandwiches - termed BUBU pulses following the nomenclature of previous J-compensated BUBI and BEBE(tr) pulse sandwiches - have been optimised.
\end{abstract}

\section{Introduction}

The emergence of cryogenically cooled probe-heads, and the historic trend of increasing magnetic field strength in NMR (nuclear magnetic resonance) spectroscopy, present new requirements for the design and implementation of effective NMR experiments. Traditional non-selective hard pulses barely cover the required bandwidths of typical heteronuclei. For example for ${ }^{13} \mathrm{C}$ spectroscopy, the uniform coverage of approximately $37.5 \mathrm{kHz}$ on a routine $600 \mathrm{MHz}$ NMR spectrometer is required, which is already a challenge. The formulation of composite pulses (Levitt, 1982) increased the pulse sequence bandwidth, covering a large resonant frequency range (Shaka and Freeman, 1983; Warren, 1984; Tycko et al., 1985: Levitt, 1986; Freeman et al. 1988). Indirectly, this success led to the development of numerical optimisation to engineer broadband composite pulses (Lurie, 1985; Conolly et al., 1986; Shaka and Pines, 1987; Emsley and Bodenhausen, 1990; Ewing et al., 1990; Garwood and $\mathrm{Ke}$, 1991). A similar development took place regarding radio-frequency pulses compensated for field inhomogeneities: when used with cryogenic probeheads with very high sensitivity to salt concentrations and typically slightly increased $\mathrm{B}_{1}$ - 
inhomogeneities, optimised pulses require an increased range of effective pulse strengths, typically covering variations in $B_{1} \geq \pm 20 \%$.

Numerically optimised pulse shapes (Glaser et al. 2015) increased the effectiveness of NMR spectroscopy with the same objective as composite pulses: tolerance to a range of pulse strengths. An explosion of pulse engineering began with the possibility to optimise pulse shapes containing thousands of independent variables, e.g. pulse amplitudes and phases (Khaneja et al. 2005). Optimal control theory has proved an indispensable tool for the optimisation of shaped pulses. At the present time, pulses are available for a variety of nuclei and bandwidths such as ${ }^{1} \mathrm{H}$ and ${ }^{13} \mathrm{C}$, but also for more specialised applications e.g. slice-selective and low-power MRI (magnetic resonance imaging) pulses (Janich et al., 2011, Vinding et al., 2012, 2017, Van Reeth et al. 2017), culminating in utmost complex pattern pulses (Kobzar et al. 2005), and hardware distorted microwave pulses for the use in EPR (electron paramagnetic resonance) spectroscopy (Spindler et al., 2012, Doll et al., 2013; Kaufmann et al. 2013, Goodwin et al. 2018). Optimal pulse engineering is leading to the question whether optimal pulses can approach the physical limit for demanding pulse robustness. Systematic studies on the optimisation of shaped pulses (Kobzar et al. 2004, 2008, 2012) and on quantum evolution with known physical limits (Reiss et al., 2002, Khaneja et al., 2002, Khaneja and Glaser. 2002, Reiss et al., 2003; , Khaneja et al., 2003b, a; (Stefanatos et al., 2004) lead to time optimal curves that are a versatile tool to find estimates for physical limits in spin dynamics (Kobzar. 2007).

As the limit of what is physically possible is neared, and control problems become computationally arduous, the development of optimal control methods is essential. There are a number of different approaches to optimal control: time optimal control (Khaneja et al. 2001); problems solved numerically with a piecewise-constant pulse approximation (Skinner et al. 2003, 2004, 2005) with linear (Khaneja et al., 2005), super-linear (de Fouquieres et al., 2011), or quadratic convergence (Goodwin and Kuprov, 2016); annealing a spin system to a desired effective Hamiltonian (Tošner et al., 2006); utilisation of cooperative multi-pulse control (Braun and Glaser, 2014); optimal control using analytic controls (Machnes et al., 2018). Specifically, the optimal control method of GRAPE (gradient ascent pulse engineering) (Khaneja et al., 2005) is used for diverse applications (Hohenester and Stadler, 2005, Palao et al., 2008, Ndong and Koch, 2010; Spindler et al., 2012; Dolde et al. 2014, Saywell et al. 2018), and can be used to construct universal rotation (UR) pulses, being unitary propagators of the system (Kobzar et al., 2012; Skinner et al., 2012; Dolde et al., 2014; Lingel et al., 2020).

The design of time optimal experiments (Khaneja et al., 2001) for time efficient coherence transfer elements (Kobzar et al. 2004, Ehni and Luy, 2012; Kobzar et al., 2012) is equally important because most of the experiment time should consist of inter-pulse delays, used for coherence transfer or chemical shift evolution, rather than radio-frequency pulsing. The simple conclusion is to ensure that total pulsing duration is as short as possible. Ideally, the total sequence duration should not be increased by the duration of the shaped pulse, $T$. This would be equivalent to the overall optimisation of the NMR experiment with respect to all relevant interactions from the underlying Hamiltonian $\mathbf{H}$, e.g. pulse strength and offset deviations of all nuclei involved in the spin system, including spin-spin coupling.

An indirect way to obtain the sequence of pulses and delays has been proposed with the ICEBERG-principle (Gershenzon et al., 2008): optimised pulse shapes followed directly by a time period of free evolution. Such pulses are named ICEBERG pulses as only a tiny fraction of the pulse contributes to the overall pulse length (in view, above the sea level), whilst the 
main part of the pulse can be considered part of the required delay time (not in view, below the sea level). These pulses have been generalised to arbitrary flip-angle RADFA pulses (Koos et al. 2015, 2017), and used e.g. in Ramsey-type cooperative experiments (Braun and Glaser, 2014). The novel feature of ICEBERG and RADFA pulses, in the context of optimal control, is that each spin in the offset ensemble has a uniquely defined terminal phase, and the ensemble produces a linear phase dispersion. In addition, UR pulses producing quadratic phase dispersion (SORDOR) have recently been proposed by Goodwin et al. (2020).

This phase is an effective $z$-rotation, and assuming radio-frequency controls dominate the Hamiltonian $\mathbf{H}$, the phase evolution can be mimicked by controls. Considering this relationship between frequency offset and controls, the ICEBERG pulses can be described as the simplest in a class of drift Hamiltonian mimicking pulses. Furthermore, setting an optimal control problem to incorporate both the shape of pulses and coherence transfer elements may reveal a path to time optimal experiments e.g. in heteronuclear spectroscopy, the effect of two concurrent UR- $180^{\circ}$ pulses set between delays is to refocus chemical shift evolution during coherence transfer (СOB-HSQC, (Ehni and Luy, 2012)) whilst retaining maximal J-evolution. This class of pulse will be termed $\mathrm{J}$-evolution, or $\mathrm{J}_{\mathrm{ev}}$, pulses in the following.

\section{Theory}

\subsection{J-Evolution and Fidelity Function}

A system irradiated in a pulsed experiment can be described as a bilinear control system,

$\mathbf{H}(t)=\mathbf{H}_{0}+\sum_{\ell}^{L} c_{\ell}(t) \mathbf{H}_{\ell}$

where $\mathbf{H}_{0}$ describes the uncontrollable parts of the system, termed the drift Hamiltonian. The controls of the system, $\mathbf{H}_{\ell}$, are associated with real-valued, time-dependent, control irradiation amplitudes, $c_{\ell}(t)$.

A system of $K$ coupled spins in isotropic solution can be described by each of their resonant frequency offsets, $\omega_{k}$, and a scalar coupling term, $J_{k, k^{\prime}}$, linking each pair of spins $k$ and $k^{\prime}$. When considering only heteronuclear systems, the weak coupling approximation can be used, which gives the drift Hamiltonian as

$\mathbf{H}_{0}=\sum_{k=1}^{K} \omega_{k} \mathbf{H}_{\mathrm{z}}^{(k)}+\sum_{k^{\prime}>k}^{K} 2 \pi J_{k, k^{\prime}}\left(\mathbf{H}_{\mathrm{z}}^{(k)} \mathbf{H}_{\mathrm{z}}^{\left(k^{\prime}\right)}\right)$

where Pauli multi-spin operators are denoted $\mathbf{H}_{\mathrm{x}, \mathrm{y}, \mathrm{z}}$.

The GRAPE method of optimal control (Khaneja et al., 2005) proceeds to describe the irradiation as piecewise constant over a small time interval $\Delta t$. This approximation allows the numerical solution of Eq. (2) with calculation of the time propagator at each time interval, $\mathbf{P}_{n}$. Consequentially, the effect of Hamiltonian dynamics from a time $t_{0}$ to a particular time $t_{n}$ is described by the time-ordered effective propagator, $\mathbf{U}_{n}$, starting from the initial propagator of the system $\mathbf{P}_{0}$, usually the identity matrix, 
and $\mathbf{V}_{n}$ are the effective propagators of the adjoint control problem, propagated with reverse time-order

$$
\begin{aligned}
\mathbf{P}_{n}=\exp \left\{-i \mathbf{H}_{n} \Delta t\right\}, & \mathbf{U}_{n}=\mathbf{P}_{n} \ldots \mathbf{P}_{1} \mathbf{P}_{0} \\
\mathbf{V}_{n} & =\mathbf{P}_{n+1}^{\dagger} \ldots \mathbf{P}_{N}^{\dagger} \mathbf{R} .
\end{aligned}
$$

J-coupling evolution can be included in the description of frequency offset and/or $B_{1}$ compensated pulses (Ehni and Luy. 2012, Kobzar et al., 2012, Ehni and Luy, 2013, 2014), mapping both into J-robustness. A measure to numerically optimise such pulses is the distance between a desired effective propagator, $\mathbf{R}$, and the implemented effective propagator, $\mathbf{U}_{N}$, using $\left\|\mathbf{R}-\mathbf{U}_{N}\right\|$ (Khaneja et al. 2005; Tošner et al. 2006), which can be expressed as the maximisation of their scalar product (Hilbert-Schmidt inner product)

$$
\max _{c_{\ell}} \Phi=\max _{c_{\ell}}\left[\operatorname{Re}\left\langle\mathbf{R} \mid \mathbf{U}_{N}\right\rangle\right]
$$

A broadband pulse sequence, additionally robust with respect to scaling of the radio-frequency amplitude, is defined by the average over a set of $n_{\text {off }}$ equally spaced offsets $\omega_{\mathrm{o}}$ and $n_{\text {rf }}$ equally spaced scaled maximum radio-frequency amplitudes $\omega_{\text {rf }}$ and $n_{J}$ equally spaced $\mathbf{J}$-couplings in the desired range of offset, radio-frequency scaling and J-coupling (Khaneja et al., 2005).

In this way, two concurrent J-compensated $180^{\circ}$ broadband UR pulses (abbreviated as BUBU-pulse - a Broadband Universal, Broadband Universal sandwich) can be found with optimal control with the desired target propagator

$$
\mathbf{R}_{\pi}=\exp \left\{-i \pi\left(\mathbf{H}_{\mathrm{x}}^{(1)}+\mathbf{H}_{\mathrm{x}}^{(2)}\right)\right\}
$$

Optimisations using $\Phi$ yield robust analogues (Fig. 1(B)) of concurrent hard $180^{\circ}$ pulses. These refocusing pulses are used in the centre of delays whereupon the total sequence duration is increased by the length of the shaped pulse $T$ (as indicated by the dotted lines in Fig. 1).

A further assertion is made: shorter sequences can be obtained when the delays are partly moved into the shaped pulse (Fig.11.C)). A fraction of time, indicated in Fig.11.C), is defined by

$$
\tau=q T, \quad q \in[0,1] .
$$

In this case, the total sequence duration would be decreased by $\tau$, and is equivalent to the idea that J-coupling evolution is mediated through the pulse. This concept is realised by a new fidelity function $\Phi_{\mathrm{ev}}$. Accordingly, pulses obtained by $\Phi_{\mathrm{ev}}$ are termed J-evolution, or simply $\mathrm{J}_{\mathrm{ev}}$, pulses.

$\Phi_{\mathrm{ev}}=\operatorname{Re}\left\langle\mathbf{R}_{\mathrm{ev}} \mid \mathbf{U}_{\mathrm{eff}}\right\rangle$

with

$$
\mathbf{R}_{\mathrm{ev}}=\mathbf{R}_{J} \mathbf{R}_{\pi} \mathbf{R}_{J}
$$

and

$$
\mathbf{R}_{J}=\exp \left\{-i 2 \pi J \mathbf{H}_{\mathrm{z}}^{(1)} \mathbf{H}_{\mathrm{z}}^{(2)} \frac{q T}{2}\right\}
$$



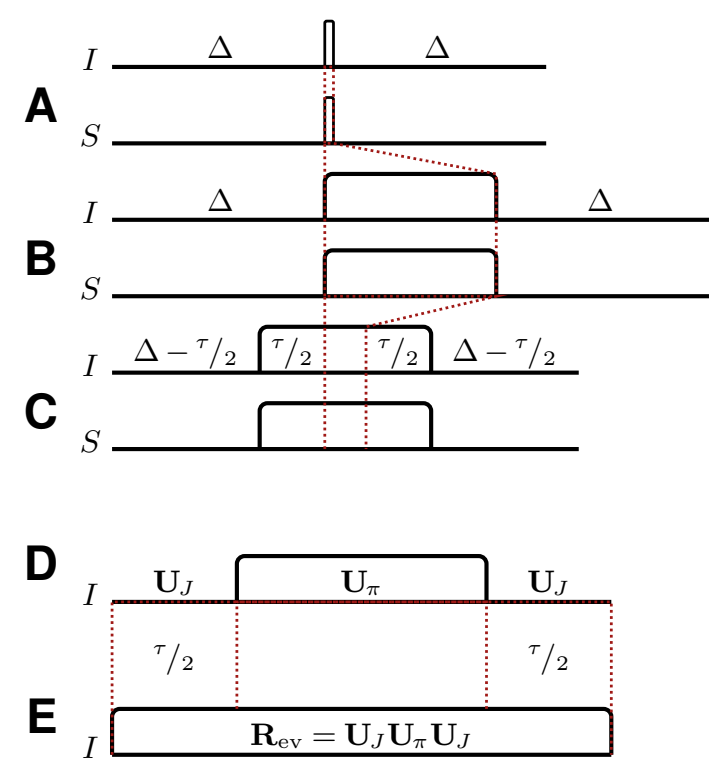

Figure 1. Spin-echo pulse sequences for the refocusing of chemical shift during heteronuclear coupling evolution with respect to coupled nuclei $H^{(k)}$ and $H^{\left(k^{\prime}\right)}$. With (A) concurrent hard pulses, (B) concurrent shaped pulses that increase the total sequence duration to $=2 \Delta+T$, (C) concurrent shaped pulses with total sequence duration decreased to $=2 \Delta+T-\tau$ that can be imagined as a delay $\tau$ that is moved inside the shaped pulse to result in a $\mathrm{J}_{\mathrm{ev}}$ pulse. (D) $\mathrm{J}_{\mathrm{ev}}$ pulses are comparable to $\mathrm{J}_{\mathrm{ev}}$-building blocks that are composed from BUBU refocusing pulses and delays $\tau$. A $\mathbf{J}_{\mathrm{ev}}$ pulse (E) is made up of the product of unitary rotations. $\mathbf{R}_{J}$ is the unitary propagator that results from a free precession period $\tau / 2$. This is combined with the effect of a refocusing pulse $\mathbf{R}_{\pi}$ to form $\mathbf{R}_{\mathrm{ev}}$ that is the final propagator of a $\mathbf{J}_{\mathrm{ev}}$ pulse.

The target propagator $\mathbf{R}_{\mathrm{ev}}$ (Fig. 1 (E)) can be written as the product of $\mathbf{R}_{J}$ and $\mathbf{R}_{\pi}$. It can be imagined as a pulse that mediates the effect of a free precession period $\tau / 2$ followed by an ideal $180^{\circ}$ concurrent refocusing pulse followed by one more $\tau / 2$ precession period as depicted in Fig. 1(D). The optimisation needs to accommodate that in a shaped pulse.

\subsection{Calculation of Gradients}

The gradient-following GRAPE method of optimal control requires directional propagator derivatives at each time increment, $D_{\sigma}\left(\mathbf{P}_{n}\right)$, in the direction of each control operator. For phase modulated pulses, the gradient vector is constructed from the elements (Skinner et al.,2006)

$\frac{\partial \Phi}{\partial \varphi_{n}}=x_{n}\left\langle\mathbf{V}_{n} \mid D_{\sigma_{\mathrm{y}}}\left(\mathbf{P}_{n}\right) \mathbf{U}_{n}\right\rangle-y_{n}\left\langle\mathbf{V}_{n} \mid D_{\sigma_{\mathrm{x}}}\left(\mathbf{P}_{n}\right) \mathbf{U}_{n}\right\rangle$

The gradient needed for the optimisation is calculated as the derivative of the fidelity function $\Phi_{\mathrm{ev}}$ with respect to the $x$ - and $y$-controls.

$\frac{\partial \Phi}{\partial c_{k}\left(t_{n}\right)}=\frac{\partial \Phi}{\partial c_{n, k}}=\operatorname{Re}\left\langle\mathbf{P}_{n+1}^{\dagger} \cdots \mathbf{P}_{N}^{\dagger} \mathbf{U}_{\text {eff }} \mid \frac{\partial \mathbf{P}_{n}}{\partial c_{n, k}} \cdots \mathbf{P}_{1}\right\rangle$ 
where the challenging task is to calculate the derivative of $\mathbf{P}$, which is given in a first order approximation by (Khaneja et al. 2005)

$\frac{\partial \Phi}{\partial c_{n, k}}=-\operatorname{Re}\left\langle\mathbf{P}_{n+1} \mid i \Delta t \mathbf{H}_{k} \mathbf{U}_{n}\right\rangle$

For the present study, however, the exact derivative of $\mathbf{P}$ is used that can be obtained, apart from other methods (Goodwin and Kuprov, 2015), by an eigenbasis transformation into the basis of the time independent Hamiltonians $\mathbf{H}$ (Levante et al. 1996).

In the basis of $\mathbf{H}$ the matrix exponential $\mathbf{P}$ and its derivatives, with respect to the controls $c_{k}$, collapse to a scalar exponential and its ordinary derivatives. Using the product rule, the derivatives of the propagator, with respect to the controls, are obtained by

$$
\begin{aligned}
\frac{\partial \mathbf{U}_{n}}{\partial c_{n, k}} & =\frac{\partial}{\partial c_{n, k}} \mathrm{e}^{-i \mathbf{H}_{n} t}=\frac{\partial}{\partial c_{n, k}} \mathbf{Q e}^{-i \boldsymbol{\Lambda}_{n} t} \mathbf{Q}^{\dagger} \\
& =\frac{\partial \mathbf{Q}}{\partial c_{n, k}} \mathrm{e}^{-i \boldsymbol{\Lambda}_{n} t} \mathbf{Q}^{\dagger}+\mathbf{Q} \frac{\partial \mathrm{e}^{-i \boldsymbol{\Lambda}_{n} t}}{\partial c_{n, k}} \mathbf{Q}^{\dagger}+\mathbf{Q} \mathrm{e}^{-i \boldsymbol{\Lambda}_{n} t} \frac{\partial \mathbf{Q}^{\dagger}}{\partial c_{n, k}} \\
& =\mathbf{Q} \frac{\partial \mathrm{e}^{-i \boldsymbol{\Lambda}_{n} t}}{\partial c_{n, k}} \mathbf{Q}^{\dagger}
\end{aligned}
$$

where $\boldsymbol{\Lambda}_{n}$ is a diagonal matrix containing the eigenvalues of $\mathbf{H}_{n}$ and $\mathbf{Q}$ is the matrix with columns of corresponding eigenvectors. The only non-zero derivative is that of the exponential function.

\subsection{Optimisation Parameters}

The initial pulse $c_{0}$ is chosen as random controls. Conjugate gradients are used for update, and controls are truncated once they exceed $\omega_{\text {rf }}=20 \mathrm{kHz}$ for ${ }^{1} \mathrm{H}$ and $\omega_{\text {rf }}=10 \mathrm{kHz}$ for ${ }^{13} \mathrm{C}$.

The optimisation parameters are chosen to accommodate the common bandwidths and $B_{1}$ field inhomogeneities for ${ }^{1} \mathrm{H}$ and ${ }^{13} \mathrm{C}$ spin systems, respectively by setting $\Delta \omega_{k}=7 \mathrm{kHz}(8), \Delta \omega_{k^{\prime}}=37.5 \mathrm{kHz}$ (42), $B_{1}$-compensations according to $\theta_{k}= \pm 20 \%$ (3), $\theta_{k^{\prime}}= \pm 5 \%$ (2), and a J-coupling up to $250 \mathrm{~Hz}$ (1). The number of explicit and equidistant evaluations of the fidelity and gradient functions are given in parentheses. Optimisation and evaluation parameters are identical except that the number of full time propagators in the respective dimension of the evaluation is somewhat higher to avoid any dependence of the fidelity function on the number of points used for evaluation.

\section{4 z-Controls}

A practical limitation of the optimisation is the large number of $\omega_{1}, \omega_{2}$, corresponding $\mathrm{B}_{1}$-inhomogeneities, J-coupling, and timesteps for a realistic pulse shape digitisation of $0.5 \mu$ s which easily results in several million propagation calculations per optimisation step. Reducing digitisation towards longer timesteps is not acceptable for the desired bandwidths: assuming a maximum carbon bandwidth of $37.5 \mathrm{kHz}$ there is a phase evolution of up to approximately $67.5^{\circ}$ within an increment duration of $\Delta t=10 \mu \mathrm{s}$. This unnecessarily brings a strong limitation to the available radio-frequency controls that can limit the fidelity obtained by a $\Phi_{\text {ev }}$-optimisation. 


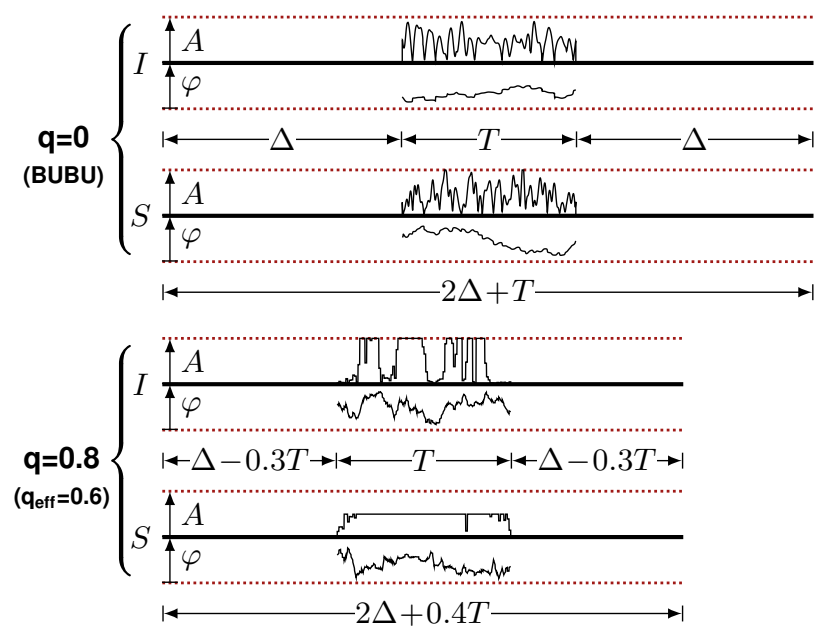

Figure 2. Amplitude, $A$, and phase, $\varphi$, of example $\mathrm{J}_{\mathrm{ev}}$ pulse sandwich with $T=1 \mathrm{~ms}$ duration, optimised for an effective $180^{\circ}$ refocusing on both $I$ and $S$ nuclei and coupling evolution according to the time fractions $q=0$ (BUBU) and $q=0.8$ (equivalent to $q$ eff $\approx 0.6$ in Eq. 15 . of Sect. 3.3. Diagrams are in the same style as Fig. 1. C). The scale of the pulse amplitude is shown with the dotted lines at $20 \mathrm{kHz}$, and the pulse phases are arbitrarily unwrapped with the scale between 0 and a maximum of $5 \times 2 \pi$ turns.

To avoid that, an additional set of controls, namely $z$-controls can be introduced. $z$-controls are calculated in accordance to the formulas derived for $x, y$-controls. $z$-controls cause $z$-rotations thereby allowing the application of frequencies with lower digitization than with conventional $x, y$-controls. They can be imagined as the effect that occurs on changing the transmitter offset to a new value. Since any $z$-rotation can be mediated also by a phase sweep of $x, y$-controls, it is possible to transform $x, y, z$-pulses into $x, y$-pulses of higher digitisation that can be realised by the available hardware. By this, the difference between high resolution $x, y$ and lower resolution $x, y, z$ pulses is reduced to a distinct incrementation of radio-frequency offset changes, while the whole range of possible radio-frequency offsets is not reduced. As such, after a pulse is obtained the $z$-controls are rendered with a resolution of $0.5 \mu$ s onto the $\Delta t=10 \mu$ s delays to obtain a pulse that can be used on the spectrometer. Nonetheless, simulations given in the following directly use the obtained $z$-controls for simplicity.

\section{Results and Discussion}

\subsection{Pulses Obtained}

Using the optimisation parameters defined in Sect.2.3, a total of five $\mathrm{J}_{\text {ev }}$ pulse sandwiches of 1 ms duration with $q \in\{0.2,0.4,0.6,0.8,0.999$ for ${ }^{1} \mathrm{H},{ }^{13} \mathrm{C}$-experiments were optimised. As an example, the BUBU pulse sandwich (with $q=0$ ) and $q=0.8$ (equivalent to $q_{\text {eff }} \approx 0.6$ in Eq. $(15)$ of Sect. 3.3) are shown in Fig. 2. The other pulses look qualitatively similar. 

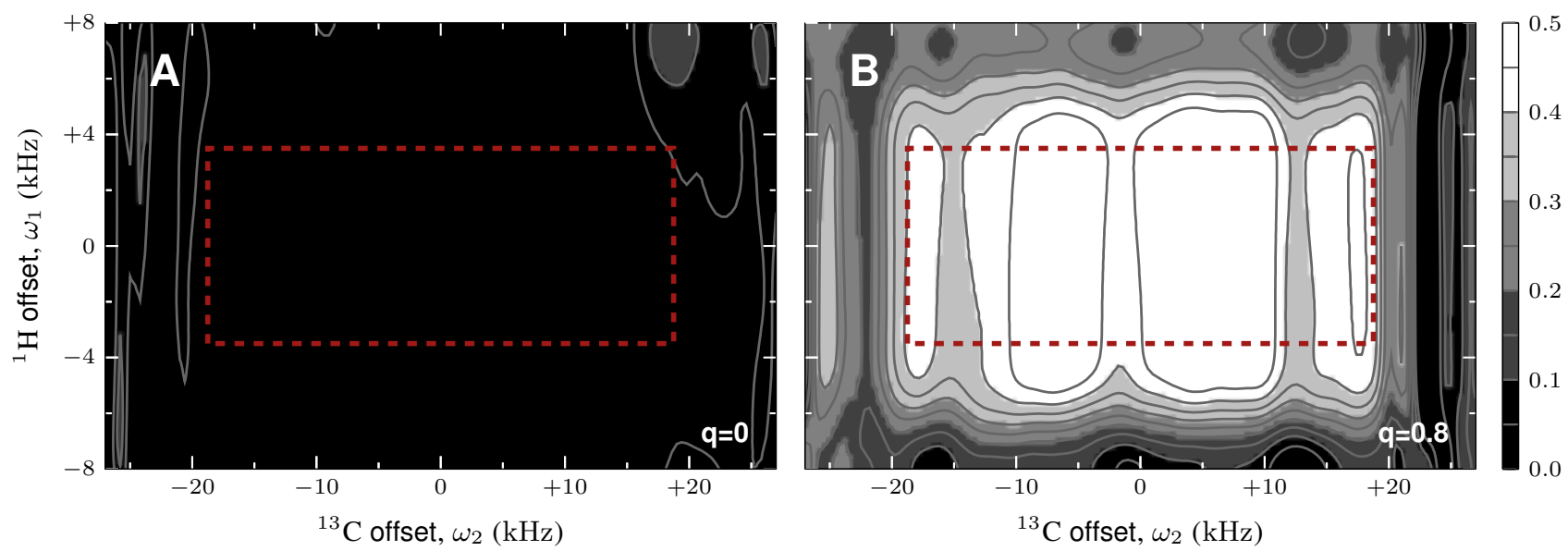

Figure 3. First order toggling frame based analysis of the scaling of heteronuclear coupling Hamiltonian with respect to ${ }^{1} \mathrm{H}$ offsets, $\omega_{1}$, and ${ }^{13} \mathrm{C}$ offsets, $\omega_{2}$. Scaling factors for optimised $\mathrm{J}_{\mathrm{ev}}$ pulse sandwiches with (A) $q=0$ (BUBU) and (B) $q=0.8$ (equivalent to $q_{\mathrm{eff}} \approx 0.6$ in Eq. (15) of Sect. 3.3, and are shown in greyscale using the scale on the right side. The dashed box indicates the region optimised for $\mathrm{J}_{\mathrm{ev}}$ pulses. A clear island of J-evolution emerges at the higher $q$ value.

As the pulses are designed to accommodate both $\mathrm{J}$-coupling evolution and a $180^{\circ}$ rotation, a proper evaluation of the $\mathrm{J}_{\mathrm{ev}}$ pulse sandwiches involve different comparisons as will be shown in the following sections.

\subsection{Detailed Evaluation of Example Pulses}

Conventionally, pulses are evaluated according to their offset dependence of transfer properties to indicate their overall performance. For the pulse sandwiches optimised here, an overall offset- and J-dependent target propagator $\mathbf{R}_{\mathrm{ev}}\left(\theta_{1}, \theta_{2}, \omega_{1}, \omega_{2}, J\right)$ is evaluated in the quality factor $\Phi_{\mathrm{ev}}$. In a first step, $\Phi_{\mathrm{ev}}$ is calculated for different offsets and $\mathrm{B}_{1}$ values: the refocusing and J-evolution is essentially fulfilled over the desired $\omega_{1}$ offset-range and $\theta_{1}$ inhomogeneity-range, which is also the case for the overall range of $\omega_{2}$ offsets, $\theta_{2}$ inhomogeneities, and J-couplings.

The direct effect from optimised pulses over the pulse duration is not useful and it should be expected that trajectories show an apparently disordered, even noisy, trajectory which starts from a defined state and finishes in a defined state, for each offset in the optimised range. This randomised feature is very different to pulse sandwiches designed by hand, as e.g. the case for selective REBURP pulses within selective INEPT steps (Lescop et al. 2010, Haller et al. 2019, Bodor et al., 2020). Nevertheless, despite the disordered behaviour, the resulting performance over the offset range is good.

Following the analysis of selective refocusing pulses in heteronuclear correlation experiments (Haller et al., 2019), also an $\left(\omega_{1}, \omega_{2}\right)$ offset dependent profile of effective J-evolution has been calculated using a toggling-frame approach with explicit inclusion of offset-frequencies. Essentially the scaling of the longitudinal heteronuclear coupling Hamiltonian $\mathbf{H}_{J}=$ $2 \pi J \mathbf{H}_{z}^{(1)} \cdot \mathbf{H}_{z}^{(2)}$ is calculated, which is directly related to the fraction $q$ of the target propagator. Unfortunately, the rela- 
tively complicated computation is beyond the scope of this publication and will be explained in detail in a different article (manuscript in preparation). As can be seen from the results shown in Fig. 3 a relatively homogeneous coupling evolution is achieved which, however, does not fully reach the fraction $q$ specified in the target propagator, indicating the physical limits of J-evolution. Fig. 3 (A) shows the coupling evolution for a low $q$ value, with no pattern and very little coupling evolution over the optimised range, which should be expected. This is in stark contrast to Fig. 3.B), for a high $q$ value, where a clear island of coupling-evolution emerges from an ocean of no coupling-evolution. A comparison of the different pulse sandwiches will be shown in the following sections using different overall fidelities to better quantify the various aspects of pulse performance.

\subsection{Overall Performance of Pulse Sandwiches}

A comparison of pulse performance for the $\mathrm{J}_{\mathrm{ev}}$ pulse sandwiches, obtained with $q \in\{0.2,0.4,0.6,0.8,0.999\}$, requires an evaluation of a fidelity metric. Here, the fidelity of Eq. (8) is initially evaluated according to Eq. (5), and is shown as a function of $q$ in Fig. 4(A-C) (dashed lines). The evaluation is done three times for different coupling constants $J \in\{145,195,250\} \mathrm{Hz}$, representing classical aliphatic as well as aromatic and triple bond scenarios. The selectivity of an arbitrary property depends on the reciprocal of the pulse length - on the order of $1 / 1 \mathrm{~ms}=1000 \mathrm{~Hz}$ for the presented $\mathrm{J}_{\mathrm{ev}}$ pulses. Accordingly, Fig. 44.A-C) (dashed lines) show a similar behaviour because the selectivity spans the whole range of coupling constants $(J \in\{145,195,250\} \mathrm{Hz})$ and approximately differ linearly as a function of the actual J-coupling constants.

Since the fidelity function $\Phi_{\mathrm{ev}}$ is intended to be a measure for both $180^{\circ}$ pulses and J-evolution, these two properties need to be resolved, in order to judge the optimised pulses, according to their J-evolution capability. As a first step we want to evaluate how far J-evolution takes place and how it contributes to the overall performance. Therefore, an ideal effective propagator $\mathbf{U}_{\mathrm{eff}, \pi}=\mathbf{R}_{\pi}$ is defined by Eq. (5) which is evaluated according to $\mathbf{R}_{\mathrm{ev}}$ in Eq. (8), using $q$ of the pulse introduced in Eq. (9), with

$\Phi_{\mathrm{ev}, \pi}=\operatorname{Re}\left\langle\mathbf{R}_{\mathrm{ev}} \mid \mathbf{U}_{\mathrm{eff}, \pi}\right\rangle$.

The numerical values of the idealised fidelity function are presented as a plot in Fig. 4(A-C) (solid line). They assign the fidelity that would be reached upon $\Phi_{\mathrm{ev}}$ by ideal concurrent $180^{\circ}$ pulses, i.e. infinitely hard pulses without radio-frequency variation. This class of pulse is not supposed to evolve J-coupling, which is the reason for the declining transfer efficiency with increasing $q$ and $J$ as illustrated in Fig. 4(A-C) (solid lines). Such ideal pulses set up a maximum fidelity that can be reached with conventional refocusing pulses. Every pulse that reports a transfer efficiency higher than this threshold must evolve J-coupling according to $\Phi_{\mathrm{ev}}$. This is observed for the discussed $\mathrm{J}_{\mathrm{ev}}$ pulses, indicating clearly that desired J-evolution during the pulse sandwich takes place. For a better quantification on the amount of overall coupling evolution, another quantity is derived by considering the threshold given by ideal $180^{\circ}$ pulses (Fig. 4(A-C), solid line): it is concluded that the range of values given by $\Phi_{\mathrm{ev}}$ is dominated by the refocusing property. Therefore, the analysis of J-coupling properties is restricted to the range of $1 \leqslant \Phi_{\mathrm{ev}, \pi}(q)$. The difference between $\Phi_{\mathrm{ev}}$ and $\Phi_{\mathrm{ev}, \pi}$ is division by $\Phi_{\mathrm{ev}, \pi}$ resulting in a fraction of the J-coupling that is acquired. This can be written as a function of $q$ to result in a constant $q_{\mathrm{eff}}$ that is actually provided by the $\mathrm{J}_{\mathrm{ev}}$ pulse according 

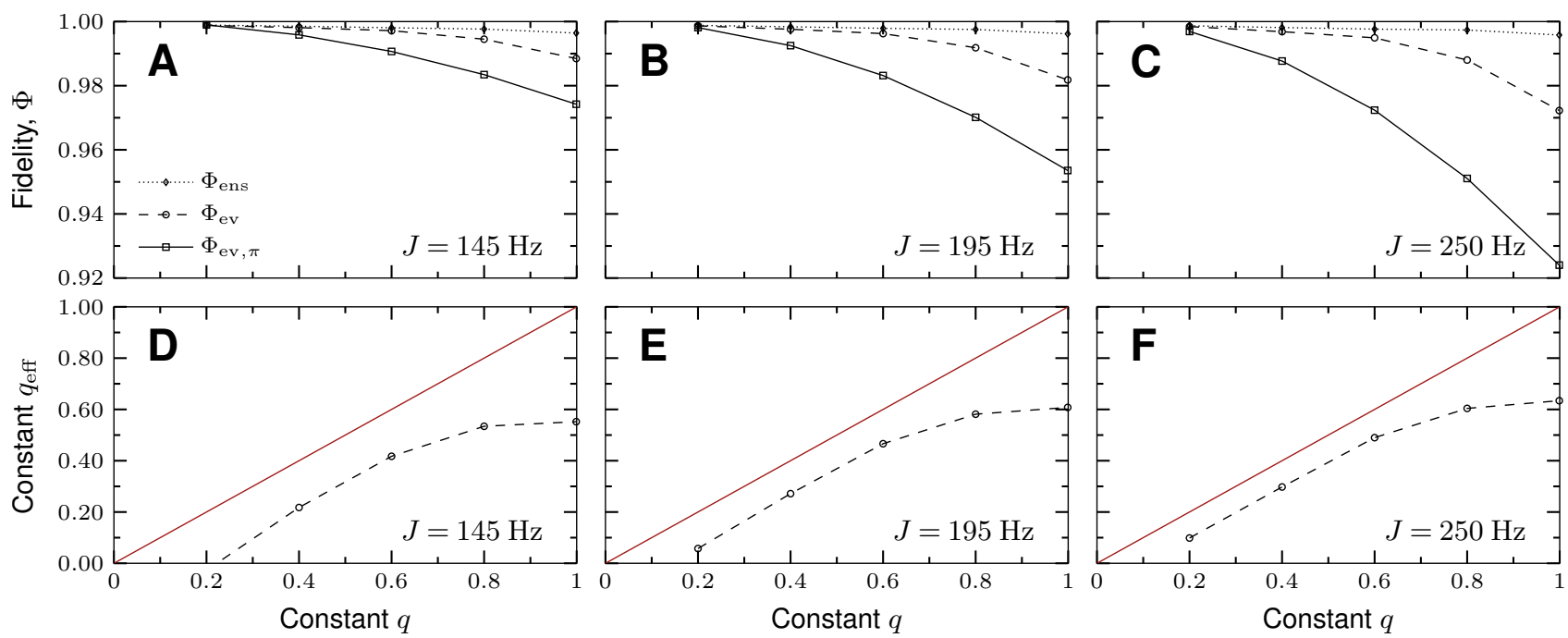

Figure 4. Transfer efficiencies for concurrent $180^{\circ}$ refocusing pulses, calculated for $J \in\{145,195,250\}$ Hz and averaged over offsets and $B_{1}$ deviations. Five $\mathrm{J}_{\mathrm{ev}}$ pulses are evaluated as a function of the J-evolution constant $q$ which demands a ratio of J-coupling that is based on the pulse length $T=1 \mathrm{~ms}$. (A-C) Upper limit of transfer efficiency that can be reached by conventional, concurrent refocusing pulses is calculated by $\Phi_{\mathrm{ev}}$ (solid lines). (A-C) $\mathrm{J}_{\mathrm{ev}}$ pulses acquire heteronuclear J-coupling and exceed that limit (dashed lines). (A-C) The $180^{\circ}$ performance of $\mathrm{J}_{\mathrm{ev}}$ pulses is obtained by the fidelity function $\Phi_{\mathrm{ens}}$ (dotted lines). (D-F) The J-coupling that has effectively been acquired upon a $\mathrm{J}_{\mathrm{ev}}$ pulse is given by $q_{\mathrm{eff}}$ (dashed lines). For $q \leqslant 0.6$ the linear behaviour and the shift according to the diagonal indicates that J-evolution performance depends on $q$.

to

$q_{\mathrm{eff}}=q \frac{\Phi_{\mathrm{ev}}-\Phi_{\mathrm{ev}, \pi}}{1-\Phi_{\mathrm{ev}, \pi}}$.

The values of $q_{\text {eff }}$ are plot as a function of $q$ in Fig. 4(D-F), and shown explicitly in Table 1 , This plot of $q_{\text {eff }}$ is nearly parallel to the diagonal for $q \lesssim 0.6$ indicating an increase of J-coupling capability as demanded by the coefficient $q$. The parallel displacement of $q_{\mathrm{eff}}$ to the diagonal is explained by the $180^{\circ}$ property of the $\mathrm{J}_{\mathrm{ev}}$ pulse sandwich: whilst the magnetisation must be in the transverse plane to acquire J-coupling, it must also leave the transverse plane to facilitate a $180^{\circ}$ rotation. The shift is caused by the fraction of time within the shaped pulse that is used for the $180^{\circ}$ rotation. With the slope of the $q_{\text {eff }}$ plot parallel to the diagonal there is an indication that there are unused resources for $q \lesssim 0.6$, which could be used for J-coupling evolution.

As there are two competing goals - the inversion on one hand and J-evolution on the other — the set goal of achieving $q$ is actually never fully fulfilled. With higher $q$ there are just more constrains on J-evolution as compared to the inversion properties. The quantity $q_{\text {eff }}$ is the amount of $q$ that you can actually get with the pulse.

Accordingly, $q \gtrsim 0.6$ assigns the region which cannot bring additional J-evolution because of the opposing requirements needed for J-evolution and $180^{\circ}$ rotations. Since J-coupling is only acquired for transverse magnetisation, it needs to leave the transverse plane for the $180^{\circ}$ rotation. Accordingly, and with the additional demand for robustness at a given pulse length 
Table 1. The constant $q_{\text {eff }}$ for a number of J-coupling values calculated using Eq. 15 for the constant $q$ from Eq. 6. from the two fidelity measures $\Phi_{\mathrm{ev}}$ and $\Phi_{\mathrm{ev}, \pi}$ from Eq. 7 and Eq. 14 respectively.

\begin{tabular}{lcccr}
\hline$q$ & $J$ & $\Phi_{\mathrm{ev}}$ & $\Phi_{\mathrm{ev}, \pi}$ & $q_{\mathrm{eff}}$ \\
\hline 0.2 & 145 & 0.9988 & 0.9990 & -0.0271 \\
0.2 & 195 & 0.9987 & 0.9981 & 0.0576 \\
0.2 & 250 & 0.9984 & 0.9969 & 0.0986 \\
0.4 & 145 & 0.9981 & 0.9959 & 0.2176 \\
0.4 & 195 & 0.9976 & 0.9925 & 0.2715 \\
0.4 & 250 & 0.9968 & 0.9877 & 0.2976 \\
0.6 & 145 & 0.9972 & 0.9907 & 0.4172 \\
0.6 & 195 & 0.9962 & 0.9832 & 0.4663 \\
0.6 & 250 & 0.9949 & 0.9724 & 0.4899 \\
0.8 & 145 & 0.9945 & 0.9834 & 0.5342 \\
0.8 & 195 & 0.9918 & 0.9701 & 0.5814 \\
0.8 & 250 & 0.9880 & 0.9511 & 0.6039 \\
0.999 & 145 & 0.9885 & 0.9742 & 0.5522 \\
0.999 & 195 & 0.9818 & 0.9535 & 0.6078 \\
0.999 & 250 & 0.9722 & 0.9240 & 0.6340 \\
\hline & & & & \\
\hline
\end{tabular}

$T=1 \mathrm{~ms}$, it is concluded that the resultant $\mathrm{J}_{\mathrm{ev}}$ pulses can acquire up to $q \approx 0.6 \mathrm{~J}$-evolution. This corresponds to $60 \%$ of $T$ and allows us to reduce the duration of flanked delays by $600 \mu$ s (shown in Fig 2).

In addition to the J-evolution capabilities of the pulse sandwich, the performance with respect to $180^{\circ}$ rotations should also be compared. Whilst the inversion of $z$-magnetisation can be calculated easily, the full rotational performance is much more difficult to characterise. For this evaluation, the quality factor $\Phi_{\mathrm{ev}}$ with $J=0 \mathrm{~Hz}$ may be used, in principle, because this implies vanishing $\mathbf{R}_{\mathrm{J}}$. However, the rotation performance will also be J-dependent, thereby masking the real performance of the pulse sandwich when only a single coupling value is used. In the authors' experience, a better definition is obtained by considering the ensemble of performances at all couplings by the fidelity function $\Phi_{\text {ens }}$ :

$$
\Phi_{\mathrm{ens}}=\max \left[\operatorname{Re}\left\langle\mathbf{R}_{\mathrm{ev}}(J) \mid \mathbf{U}_{\mathrm{eff}}\right\rangle\right], \quad J \in(0,250] .
$$

Similar to $\Phi_{\mathrm{ev}}, \Phi_{\mathrm{ens}}$ is defined with the target propagator $\mathbf{R}_{J}$ in Eq. 9 but with a difference that $J$ is not set to $J_{\max }$, e.g. $J_{\max }=195 \mathrm{~Hz}$ ), but is varied in the range of $J=\left\{0, \ldots, J_{\max }\right\}$ (with a typical increment of $\Delta J=1 \mathrm{~Hz}$ ). $\Phi_{\text {ens }}$ is calculated in that range for every combination of constraints with only the best transfer efficiency from the range $J=\left\{0, \ldots, J_{\max }\right\}$ taken for the accumulation of the final transfer efficiency. This procedure is equivalent to allowing every possible J-evolution and, essentially, monitoring the effect of the $180^{\circ}$ rotation. The resulting plot is given in Fig. 4(A-C) (dotted lines) and is shown 
with more detail in Fig. 5 (dotted line). As a general observation, the rotational performances over all $\mathrm{J}_{\mathrm{ev}}$ pulse sandwiches are good, with a small decrease in fidelity at larger $q$.

\subsection{Comparison to Pulse Sandwiches without J-Evolution}

Analysis so far has shown that it is possible to obtain $\mathrm{J}_{\mathrm{ev}}$ pulse sandwiches that acquire $\mathrm{J}$-coupling on top of a $180^{\circ}$ rotation. However, it is also necessary to also evaluate whether shorter, more conventional, $180^{\circ}$ shaped pulses, flanked by appropriate delays, are more efficient when compared to the proposed $\mathrm{J}_{\mathrm{ev}}$ pulses.

According to Fig. 1 D), any $\mathrm{J}_{\mathrm{ev}}$ pulse can be imagined as a concatenation of free evolution periods, $\tau$, with a centred, concurrent $180^{\circ}$ pulse sandwich (which we call a BUBU-pulse sandwich, following the nomenclature introduced in (Ehni and Luy, 2013), for Broadband Universal rotation pulses on both nuclei ${ }^{1} \mathrm{H}$ and ${ }^{13} \mathrm{C}$ ). Indeed, conventional shaped pulses, flanked with delays $\tau$, can be used to give $\mathrm{J}_{\mathrm{ev}}$-building blocks with duration of $1 \mathrm{~ms}$, which can be compared with the $\mathrm{J}_{\mathrm{ev}}$ pulse sandwich.

The most direct comparison between $\mathrm{J}_{\mathrm{ev}}$ pulse sandwiches and corresponding $\mathrm{J}_{\mathrm{ev}}$-building blocks (composed of two delays $\tau$ surrounding a BUBU-pulse sandwich) can be obtained by considering the $180^{\circ}$ rotation capabilities and using $\Phi_{\text {ens }}$ in Eq. (16). By definition, the $\mathrm{J}_{\mathrm{ev}}$-building block acquires the same amount of J-coupling compared to a $\mathrm{J}_{\mathrm{ev}}$ pulse defined with a given $q$. The analysis starts by finding appropriate delays $\tau$ that correspond to the J-evolution acquired upon implementation of a $\mathbf{J}_{\mathrm{ev}}$ pulse. $\tau$ is calculated starting from a given $q_{\text {eff }}$. The $q_{\text {eff }}$ values from Fig. 4(E) (dashed line) are given in Table 1 (for example $\left.q_{\mathrm{eff}}=0.6078=2 \tau\right)$. This results in $T_{\mathrm{BUBU}}=T_{J_{\mathrm{ev}}}-2 \tau=1 \mathrm{~ms}-0.6078 \mathrm{~ms}=0.3922 \mathrm{~ms}$.

In total, five BUBU-pulses are optimised for $J=195 \mathrm{~Hz}$, with durations of $T_{\mathrm{BUBU}} \in\{0.94,0.73,0.53,0.42,0.39\}$ ms have been optimised corresponding to $q_{\mathrm{eff}}=\{0.2,0.4,0.6,0.8,0.999\}$. The decreased duration of pulses allows pulses to be optimised using $x-, y$-controls with a digitisation of $0.5 \mu$ s, instead of $x-, y$-, $z$-controls, further opening the available optimisation space.

The fidelity of the BUBU-pulses is obtained according to $\Phi_{\text {ens }}$ in Eq. 16 and results are shown in Fig. 5 (solid line). This plot determines the physical threshold for concurrent $180^{\circ}$ refocusing pulses with respect to the given pulse lengths $T_{\mathrm{BUBU}}$ and required robustness. Concerning Fig. 5, the $180^{\circ}$ rotation capability of the $\mathrm{J}_{\mathrm{ev}}$ pulse sandwich (dotted line) exceeds in all cases the performance of the BUBU-pulse sandwich (solid line). Since the setup is chosen in order to start from identical amounts of $\mathrm{J}$-evolution, it is concluded that $\mathrm{J}_{\mathrm{ev}}$ pulses are more efficient compared to an equivalent $\mathrm{J}_{\mathrm{ev}}$-building block made up from BUBU-pulses and delays $\tau$. Fig. 5 shows that the efficiencies of the $\mathrm{J}_{\mathrm{ev}}$ pulse sandwiches, compared to the BUBU analogue, have largest gains with increasing $q$.

\section{Conclusions}

The $\mathrm{J}_{\mathrm{ev}}$ pulses are non-conventional pulse sandwiches that exceed the class of single spin shaped pulses. They are designed for chemical shift refocusing in heteronuclear coherence transfer elements. 


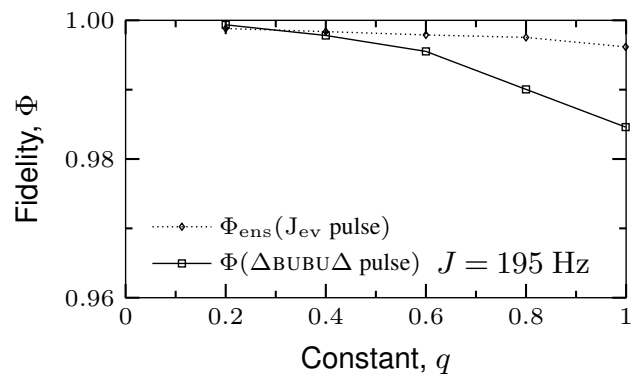

Figure 5. Transfer efficiencies for concurrent $180^{\circ}$ refocusing pulses, calculated for $J=195 \mathrm{~Hz}$ and averaged over offsets and $B_{1}$ deviations. To determine whether $\mathrm{J}_{\mathrm{ev}}$ pulse sandwiches exceed the performance of comparable $\left(\tau-180^{\circ}-\tau\right) \mathrm{J}_{\mathrm{ev}}$-building blocks (according to the relation in Fig. 1 D)), they are compared in terms of their $180^{\circ}$ fidelity. Five $\mathrm{J}_{\mathrm{ev}}$ pulses for $0.2 \leqslant q \leqslant 0.999$ are evaluated according to $\Phi_{\text {ens }}$ (dotted line) and compared to $\mathrm{J}_{\mathrm{ev}}$-building blocks derived from shorter BUBU-pulses. In all cases $\mathrm{J}_{\mathrm{ev}}$ pulse sandwiches exceed the transfer properties of BUBU-pulse sandwiches, demonstrating the gain of the new class of pulses which extend the ICEBERG-principle (Gershenzon et al. 2008).

Usually, delays dominate the length of typical coherence transfer elements (e.g. that of the INEPT (Morris and Freeman, 1979) or COB-INEPT (Ehni and Luy, 2012)) and thereby also the total sequence duration. In order to obtain time optimal coherence transfers, it is not necessary to optimise the sequence as a whole. A fraction of the delay might be located inside the refocusing pulses, reducing the sequence length to a total duration which is closer to time-optimal.

For this reason a concept is derived from these so called $\mathrm{J}_{\mathrm{ev}}$ pulses, with the example of J-evolved BUBU-pulses, in that they have good refocusing properties on both nuclei, ${ }^{1} \mathrm{H}$ and ${ }^{13} \mathrm{C}$, and also evolve J-coupling on top of these refocusing properties.

A strategy to analyse the obtained pulses is described based on the decomposition of the mutual refocusing and J-evolution properties. It is clear that the proposed $\mathrm{J}_{\mathrm{ev}}$ pulses facilitate $\mathrm{J}$-coupling evolution.

Using a second type of decomposition strategy and a set of comparable, conventional pulses, it is shown that $\mathrm{J}_{\mathrm{ev}}$ pulses not only evolve J-coupling but they also result in sequences with reduced overall time consumption which is inaccessible with conventional pulses, and therefore closer to the physical limit of pulse sequence duration. The advantage in time consumption can be given as a fraction of the $\mathrm{J}_{\mathrm{ev}}$ pulse length $T=1 \mathrm{~ms}$ and is found to be approximately $q_{\mathrm{eff}}=0.6$ for the studied set of constraints, resulting in a reduced time demand of $0.6 \mathrm{~ms}$ for every $\mathrm{J}_{\mathrm{ev}}$ pulse that is applied in a sequence, while maintaining the benefits from offset- and $B_{1}$-compensated pulse shapes.

Acknowledgements. This project is funded by the Helmholtz programme BIFTM (47.02.04) and Information (43.35.02) and the Deutsche Forschungsgemeinschaft (DFG LU 835/11-1 and LU 835/13-1). 


\section{References}

Bodor, A., Haller, J. D., Bouguechtouli, C., Theillet, F.-X., Nyitray, L., and Luy, B.: Power of Pure Shift H $\alpha$ C $\alpha$ Correlations: A Way to Characterize Biomolecules under Physiological Conditions, Anal. Chem., 92, 12 423-12 428, https://doi.org/10.1021/acs.analchem.0c02182 2020.

Braun, M. and Glaser, S. J.: Concurrently optimized cooperative pulses in robust quantum control: application to broadband Ramsey-type pulse sequence elements, New J. Phys., 16, 115 002, https://doi.org/10.1088/1367-2630/16/11/115002 2014.

Conolly, S., Nishimura, D., and Macovski, A.: Optimal control solutions to the magnetic resonance selective excitation problem, IEEE Trans. Med. Imag., 5, 106-115, https://doi.org/10.1109/tmi.1986.4307754 1986.

de Fouquieres, P., Schirmer, S. G., Glaser, S. J., and Kuprov, I.: Second order gradient ascent pulse engineering, J. Magn. Reson., 212, 412-417, https://doi.org/10.1016/j.jmr.2011.07.023 2011.

Dolde, F., Bergholm, V., Wang, Y., Jakobi, I., Naydenov, B., Pezzagna, S., Meijer, J., Jelezko, F., Neumann, P., SchulteHerbrüggen, T., Biamonte, J., and Wrachtrup, J.: High-fidelity spin entanglement using optimal control, Nat. Commun., 5, 3371, https://doi.org/10.1038/ncomms4371, 2014.

Doll, A., Pribitzer, S., Tschaggelar, R., and Jeschke, G.: Adiabatic and fast passage ultra-wideband inversion in pulsed EPR, J. Magn. Reson., 230, 27-39, https://doi.org/10.1016/j.jmr.2013.01.002, 2013.

Ehni, S. and Luy, B.: A systematic approach for optimizing the robustness of pulse sequence elements with respect to couplings, offsets, and B1-field inhomogeneities (COB), Magn. Reson. Chem., 50, 63-72, https://doi.org/10.1002/mrc.3846, 2012.

Ehni, S. and Luy, B.: BEBE ${ }^{\text {tr }}$ and BUBI: J-compensated concurrent shaped pulses for 1H-13C experiments, J. Magn. Reson., 232, 7-17, https://doi.org/10.1016/j.jmr.2013.04.007, 2013.

Ehni, S. and Luy, B.: Robust INEPT and refocused INEPT transfer with compensation of a wide range of couplings, offsets, and B1-field inhomogeneities (COB3), J. Magn. Reson., 247, 111-117, https://doi.org/10.1016/j.jmr.2014.07.010, 2014.

Emsley, L. and Bodenhausen, G.: Gaussian pulse cascades: New analytical functions for rectangular selective inversion and in-phase excitation in NMR, Chem. Phys. Lett., 165, 469-476, https://doi.org/10.1016/0009-2614(90)87025-m 1990.

Ewing, B., Glaser, S. J., and Drobny, G. P.: Development and optimization of shaped NMR pulses for the study of coupled spin systems, J. Chem. Phys., 147, 121-129, https://doi.org/10.1016/0301-0104(90)85028-u 1990.

Freeman, R., Friedrich, J., and Xi-li, W.: A pulse for all seasons. Fourier transform spectra without a phase gradient, J. Magn. Reson. (1969), 79, 561-567, https://doi.org/10.1016/0022-2364(88)90092-3, 1988.

Garwood, M. and Ke, Y.: Symmetric pulses to induce arbitrary flip angles with compensation for rf inhomogeneity and resonance offsets, J. Magn. Reson. (1969), 94, 511-525, https://doi.org/10.1016/0022-2364(91)90137-i. 1991.

Gershenzon, N. I., Skinner, T. E., Brutscher, B., Khaneja, N., Nimbalkar, M., Luy, B., and Glaser, S. J.: Linear phase slope in pulse design: Application to coherence transfer, J. Magn. Reson., 192, 235-243, https://doi.org/10.1016/j.jmr.2008.02.021. 2008.

Glaser, S. J., Boscain, U., Calarco, T., Koch, C. P., Köckenberger, W., Kosloff, R., Kuprov, I., Luy, B., Schirmer, S., SchulteHerbrüggen, T., Sugny, D., and Wilhelm, F. K.: Training Schrödinger's cat: quantum optimal control, Eur. Phys. J. D, 69, 279, https://doi.org/10.1140/epjd/e2015-60464-1, 2015.

Goodwin, D. L. and Kuprov, I.: Auxiliary matrix formalism for interaction representation transformations, optimal control, and spin relaxation theories, J. Chem. Phys., 143, 084 113, https://doi.org/10.1063/1.4928978, 2015. 
Goodwin, D. L. and Kuprov, I.: Modified Newton-Raphson GRAPE methods for optimal control of spin systems, J. Chem. Phys., 144, 204 107, https://doi.org/10.1063/1.4949534, 2016.

Goodwin, D. L., Myers, W. K., Timmel, C. R., and Kuprov, I.: Feedback control optimisation of ESR experiments, J. Magn. Reson., 297, 9-16, https://doi.org/10.1016/j.jmr.2018.09.009 2018.

Goodwin, D. L., Koos, M. R. M., and Luy, B.: Second Order Phase Dispersion by Optimised Rotations, Phys. Rev. Res., 2, 033 157, https://doi.org/10.1103/PhysRevResearch.2.033157, 2020.

Haller, J. D., Bodor, A., and Luy, B.: Real-time pure shift measurements for uniformly isotope-labeled molecules using X-selective BIRD homonuclear decoupling, J. Magn. Reson., 302, 64-71, https://doi.org/10.1016/j.jmr.2019.03.011, 2019.

Hohenester, U. and Stadler, G.: Optimal quantum control of electron-phonon scatterings in artificial atoms, Physica E, 29, 320, https://doi.org/10.1016/j.physe.2005.05.029, 2005.

Janich, M. A., Schulte, R. F., Schwaiger, M., and Glaser, S. J.: Robust slice-selective broadband refocusing pulses, J. Magn. Reson., 213, 126-135, https://doi.org/10.1016/j.jmr.2011.09.025 2011.

Kaufmann, T., Keller, T. J., Franck, J. M., Barnes, R. P., Glaser, S. J., Martinis, J. M., and Han, S.: DAC-board based X-band EPR spectrometer with arbitrary waveform control, J. Magn. Reson., 235, 95-108, https://doi.org/10.1016/j.jmr.2013.07.015. 2013.

Khaneja, N. and Glaser, S. J.: Efficient transfer of coherence through Ising spin chains, Phys. Rev. A, 66, 060301, https://doi.org/10.1103/physreva.66.060301, 2002.

Khaneja, N., Brockett, R., and Glaser, S. J.: Time optimal control in spin systems, Phys. Rev. A, 63, 032308, https://doi.org/10.1103/physreva.63.032308, 2001.

Khaneja, N., Glaser, S. J., and Brockett, R.: Sub-Riemannian geometry and time optimal control of three spin systems: Quantum gates and coherence transfer, Phys. Rev. A, 65, https://doi.org/10.1103/physreva.65.032301, 2002.

Khaneja, N., Luy, B., and Glaser, S. J.: Boundary of quantum evolution under decoherence, Proc. Natl. Acad. Sci., 100, 13 162-13 166, https://doi.org/10.1073/pnas.2134111100, 2003a.

Khaneja, N., Reiss, T., Luy, B., and Glaser, S. J.: Optimal control of spin dynamics in the presence of relaxation, J. Magn. Reson., 162, 311-319, https://doi.org/10.1016/s1090-7807(03)00003-x 2003b.

Khaneja, N., Reiss, T., Kehlet, C., Schulte-Herbrüggen, T., and Glaser, S. J.: Optimal control of coupled spin dynamics: design of NMR pulse sequences by gradient ascent algorithms, J. Magn. Reson., 172, 296-305, https://doi.org/10.1016/j.jmr.2004.11.004 2005.

Kobzar, K.: Optimal Control, Partial Alignment and More: The Design Of Novel Tools for NMR Spectroscopy of Small Molecules, Ph.D. thesis, Technische Universität München, 2007.

Kobzar, K., Skinner, T. E., Khaneja, N., Glaser, S. J., and Luy, B.: Exploring the limits of broadband excitation and inversion pulses, J. Magn. Reson., 170, 236-243, https://doi.org/10.1016/j.jmr.2004.06.017. 2004.

Kobzar, K., Luy, B., Khaneja, N., and Glaser, S. J.: Pattern pulses: design of arbitrary excitation profiles as a function of pulse amplitude and offset, J. Magn. Reson., 173, 229-235, https://doi.org/10.1016/j.jmr.2004.12.005 2005.

Kobzar, K., Skinner, T. E., Khaneja, N., Glaser, S. J., and Luy, B.: Exploring the limits of broadband excitation and inversion: II. Rf-power optimized pulses, J. Magn. Reson., 194, 58-66, https://doi.org/10.1016/j.jmr.2008.05.023 2008.

Kobzar, K., Ehni, S., Skinner, T. E., Glaser, S. J., and Luy, B.: Exploring the limits of broadband $90^{\circ}$ and $180^{\circ}$ universal rotation pulses, J. Magn. Reson., 225, 142-160, https://doi.org/10.1016/j.jmr.2012.09.013, 2012.

Koos, M. R. M., Feyrer, H., and Luy, B.: Broadband excitation pulses with variable RF amplitude-dependent flip angle (RADFA), Magn. Reson. Chem., 53, 886-893, https://doi.org/10.1002/mrc.4297| 2015. 
Koos, M. R. M., Feyrer, H., and Luy, B.: Broadband RF-amplitude-dependent flip angle pulses with linear phase slope, Magn. Reson. Chem., 55, 797-803, https://doi.org/10.1002/mrc.4593, 2017.

Lescop, E., Kern, T., and Brutscher, B.: Guidelines for the use of band-selective radiofrequency pulses in hetero-nuclear NMR: example of longitudinal-relaxation-enhanced BEST-type 1H-15N correlation experiments, J. Magn. Reson., 203, 190-198, https://doi.org/10.1016/j.jmr.2009.12.001, 2010.

Levante, T. O., Bremi, T., and Ernst, R. R.: Pulse-sequence optimization with analytical derivatives. Application to deuterium decoupling in oriented phases, J. Magn. Reson., A, 121, 167-177, https://doi.org/10.1006/jmra.1996.0157. 1996.

Levitt, M. H.: Symmetrical composite pulse sequences for NMR population inversion. I. Compensation of radiofrequency field inhomogeneity, J. Magn. Reson. (1969), 48, 234-264, https://doi.org/10.1016/0022-2364(82)90275-x 1982.

Levitt, M. H.: Composite Pulses, Prog. Nucl. Magn. Reson. Spectrosc., 18, 61-122, https://doi.org/10.1016/0079-6565(86)80005-X 1986.

Lingel, A., Vulpetti, A., Reinsperger, T., Proudfoot, A., Denay, R., Frommlet, A., Henry, C., Hommel, U., Gossert, A. D., Luy, B., and Frank, A. O.: Comprehensive and High-Throughput Exploration of Chemical Space Using Broadband 19F NMR-Based Screening, Angew. Chem., 59, 14 809-14 817, https://doi.org/10.1002/anie.202002463. 2020.

Lurie, D. J.: A systematic design procedure for selective pulses in NMR imaging, Magn. Reson. Imag., 3, 235-243, https://doi.org/10.1016/0730-725x(85)90352-2, 1985.

Machnes, S., Assémat, E., Tannor, D., and Wilhelm, F. K.: Tunable, Flexible, and Efficient Optimization of Control Pulses for Practical Qubits, Phys. Rev. Lett., 120, 150 401, https://doi.org/10.1103/physrevlett.120.150401, 2018.

Morris, G. A. and Freeman, R.: Enhancement of nuclear magnetic resonance signals by polarization transfer, J. Am. Chem. Soc., 101, 760, https://doi.org/10.1021/ja00497a058 1979.

Ndong, M. and Koch, C. P.: Vibrational stabilization of ultracold KRb molecules: A comparative study, Phys. Rev. A, 83, 43437, https://doi.org/10.1103/PhysRevA.82.043437, 2010.

Palao, J. P., Kosloff, R., and Koch, C. P.: Protecting coherence in optimal control theory: State-dependent constraint approach, Phys. Rev. A, 77, 63 412, https://doi.org/10.1103/PhysRevA.77.063412, 2008.

Reiss, T. O., Khaneja, N., and Glaser, S. J.: Time-Optimal Coherence-Order-Selective Transfer of In-Phase Coherence in Heteronuclear IS Spin Systems, J. Magn. Reson., 154, 192-195, https://doi.org/10.1006/jmre.2001.2480, 2002.

Reiss, T. O., Khaneja, N., and Glaser, S. J.: Broadband geodesic pulses for three spin systems: time-optimal realization of effective trilinear coupling terms and indirect SWAP gates, J. Magn. Reson., 165, 95-101, https://doi.org/10.1016/s1090-7807(03)00245-3. 2003.

Saywell, J. C., Kuprov, I., Goodwin, D., Carey, M., and Freegarde, T.: Optimal control of mirror pulses for cold-atom interferometry, Phys. Rev. A, 98, 023 625, https://doi.org/10.1103/physreva.98.023625, 2018.

Shaka, A. and Freeman, R.: Composite pulses with dual compensation, J. Magn. Reson. (1969), 55, 487-493, https://doi.org/10.1016/00222364(83)90133-6. 1983.

Shaka, A. and Pines, A.: Symmetric phase-alternating composite pulses, J. Magn. Reson. (1969), 71, 495-503, https://doi.org/10.1016/00222364(87)90249-6, 1987.

Skinner, T. E., Reiss, T. O., Luy, B., Khaneja, N., and Glaser, S. J.: Application of optimal control theory to the design of broadband excitation pulses for high-resolution NMR, J. Magn. Reson., 163, 8-15, https://doi.org/10.1016/S1090-7807(03)00153-8. 2003.

Skinner, T. E., Reiss, T. O., Luy, B., Khaneja, N., and Glaser, S. J.: Reducing the duration of broadband excitation pulses using optimal control with limited RF amplitude, J. Magn. Reson., 167, 68-74, https://doi.org/10.1016/j.jmr.2003.12.001, 2004. 
Skinner, T. E., Reiss, T. O., Luy, B., Khaneja, N., and Glaser, S. J.: Tailoring the optimal control cost function to a desired output: application to minimizing phase errors in short broadband excitation pulses, J. Magn. Reson., 172, 17-23, https://doi.org/10.1016/j.jmr.2004.09.011 2005.

Skinner, T. E., Kobzar, K., Luy, B., Bendall, M. R., Bermel, W., Khaneja, N., and Glaser, S. J.: Optimal control design of constant amplitude phase-modulated pulses: Application to calibration-free broadband excitation, J. Magn. Reson., 179, 241-249, https://doi.org/10.1016/j.jmr.2005.12.010, 2006.

Skinner, T. E., Gershenzon, N. I., Nimbalkar, M., Bermel, W., Luy, B., and Glaser, S. J.: New strategies for designing robust universal rotation pulses: Application to broadband refocusing at low power, J. Magn. Reson., 216, 78-87, https://doi.org/10.1016/j.jmr.2012.01.005. 2012.

Spindler, P. E., Zhang, Y., Endeward, B., Gershernzon, N., Skinner, T. E., Glaser, S. J., and Prisner, T. F.: Shaped optimal control pulses for increased excitation bandwidth in EPR, J. Magn. Reson., 218, 49-58, https://doi.org/10.1016/j.jmr.2012.02.013, 2012.

Stefanatos, D., Khaneja, N., and Glaser, S. J.: Optimal control of coupled spins in the presence of longitudinal and transverse relaxation, Phys. Rev. A, 69, 022 319, https://doi.org/10.1103/physreva.69.022319, 2004.

Tošner, Z., Glaser, S. J., Khaneja, N., and Nielsen, N. C.: Effective Hamiltonians by optimal control: Solid-state NMR double-quantum planar and isotropic dipolar recoupling, J. Chem. Phys., 125, 184 502, https://doi.org/10.1063/1.2366703. 2006.

Tycko, R., Cho, H., Schneider, E., and Pines, A.: Composite pulses without phase distortion, J. Magn. Reson. (1969), 61, 90-101, https://doi.org/10.1016/0022-2364(85)90270-7, 1985.

Van Reeth, E., Ratiney, H., Tesch, M., Grenier, D., Beuf, O., Glaser, S. J., and Sugny, D.: Optimal control design of preparation pulses for contrast optimization in MRI, J. Magn. Reson., 279, 39-50, https://doi.org/10.1016/j.jmr.2017.04.012 2017.

Vinding, M. S., Maximov, I. I., Tošner, Z., and Nielsen, N. C.: Fast numerical design of spatial-selective rf pulses in MRI using Krotov and quasi-Newton based optimal control methods, J. Chem. Phys., 137, 054 203, https://doi.org/10.1063/1.4739755. 2012.

Vinding, M. S., Brenner, D., Tse, D. H. Y., Vellmer, S., Vosegaard, T., Suter, D., Stöcker, T., and Maximov, I. I.: Application of the limited-memory quasi-Newton algorithm for multi-dimensional, large flip-angle RF pulses at 7T, Magn. Reson. Mater. Phy., 30, 29-39, https://doi.org/10.1007/s10334-016-0580-1, 2017.

Warren, W. S.: Effects of arbitrary laser or NMR pulse shapes on population inversion and coherence, J. Chem. Phys., 81, 5437-5448, https://doi.org/10.1063/1.447644, 1984. 